\title{
Non-Conventional Standpoint on Spin Crossovers in the Organometallic Compounds of Iron Group
}

\author{
V. V. Shelest ${ }^{1}$, A. V. Hristov ${ }^{2}$, A. Yu. Prokhorov ${ }^{2}$, D. A. Chervinskii ${ }^{1}$ \\ ${ }^{1}$ Dept. of Dynamical Properties of Complex Systems, Galkin Institute for Physics \& Engineering, Donetsk, Ukraine \\ ${ }^{2}$ Dept. of Phase Transitions, Galkin Institute for Physics \& Engineering, Donetsk, Ukraine
}

Email address:

chervin@teor.fti.ac.donetsk.ua (D. A. Chervinskii), efbji@list.ru (A. V. Hristov)

\section{To cite this article:}

V. V. Shelest, A. V. Hristov, A. Yu. Prokhorov, D. A. Chervinskii. Non-conventional Standpoint on Spin Crossovers in the Organometallic Compounds of Iron Group. Journal of Photonic Materials and Technology. Vol. 1, No. 2, 2015, pp. 40-45. doi: 10.11648/j.jmpt.20150102.14

\begin{abstract}
We have considered the spin crossover (SC) complex compounds containing spin-active metal ions of iron group, which can induce the spin transitions (ST) of high spin (HS) - low spin (LS) type under influence of temperature (TIST), pressure (PIST) or light (LIESST) from non-conventional point of view. We have analyzed the physical peculiarities of the studied ST systems observed in numerous papers for X ray, spectral, calorimetric and magnetic measurements. On this basis, we suppose that ST (for example, in bulk of SC compounds with TIST) can be considered as phase transitions (PT) of postcritical type (PCT).
\end{abstract}

Keywords: Spin Crossover, Phase Transition, Critical Phenomena, Supramolecular Compounds

\section{Introduction}

Temperature-, pressure- and light-induced (TIST, PIST, LIESST) spin transitions (ST) or spin crossovers (SC) are one of the most fascinating dynamic electronic structure phenomena occurring in coordination compounds of transition metal ions, mostly iron(II), cobalt(II), iron(III). These crossovers are attractive for many research groups all over the world [1-10 (ref. ibid)]. For example, spin state of certain octahedral $d^{6}$ iron (II) complexes may be switched between low spin (LS) and high spin (HS) states with nearly equal energies (LS: $S=0, \mathrm{t}_{2 \mathrm{~g}}{ }^{6} \mathrm{e}_{\mathrm{g}}$; HS: $S=2, \mathrm{t}_{2 \mathrm{~g}}{ }^{4} \mathrm{e}_{\mathrm{g}}{ }^{2}$ ). Collective behavior of such bistable molecules forming a crystal lattice can lead to multistability in solid state described by complex temperature and pressure dependencies. Such phenomena are not only of great interest for fundamental science but also hold a large potential for technological applications [4-10].

\section{Micro- and Macroaspects of SC}

Fundamental interest to the phenomenon is aimed on deeper studying of its mechanism. Nature of this mechanism in supramolecular systems is unique. This is a challenge for scientists, surprising them for any concrete case of ST inducing by features of the forming and evolution of the spin states. For any special case of system response on external disturbing factors such as temperature, pressure, light, magnetic field reveals the basic physical principles for ST formation.

One of the main fundamental principles for ST formation is a relationship between the spin states (LS, HS) of spincrossover metal ion, effective size of its shell for a certain spin state and the environment volume. This dependence is caused, on the one hand, by chemical structure of elements forming a crystal, in the first instance, spin-crossover metal ion and ligands of the first coordination sphere. On the other hand, spin state of the metal ion is determined by static deformations due to the packing effect, internal (chemical) pressure and elastic properties of the environment. Note that for any case of ST, inducing the deformations, always are present deformations which stipulate a variation of angular coordinates of the system changing the form of given volume. These deformations coexist together with the static-dynamic deformations SC systems which are expressed through the changes of bond length and, finally, displayed the change of specific volume of the compound. Both certainly play an important role in process of spin state formation in the system, and indeed, relate (directly or indirectly) to ST including the hysteresis phenomena. It is obvious that the role of the deformation changing the form of its volume is more significant for PIST and LIST. It is especially urgent taking into account for observed low symmetry of manymolecular molecules-clusters including, for example, 
pseudo-octahedrons and other strained complexes. [5-11 and ref. ibid].

In view of the fact the $\mathrm{X}$-ray experiments give the changes of bond length for metal-ligand near 0.2 $\AA$ [4-10 and ref. ibid]. That is quite appreciable value for TIST and LIST (low temperatures). Due to characteristic anisotropy of ST system this causes a significant change of angular variables of the couplings under influence of external disturbances. It is assumed that above-mentioned dependence of HS and LS states is connected only with structure features of unfilled valence $3 d$ shell of metal ion and its environment. The hysteresis is usually related to cooperative behavior of the couplings between spin-crossover ions interacting by elastic mode and to energy losses at overcoming a barrier between HS and LS states.

In general, X-ray spectroscopy indicates on low symmetry of the ST systems [4-10, 11 (ref. ibid)]. Infrared and Raman spectroscopy, nuclear inelastic scattering and DFT calculations show the connection between ST and anharmonicity [4-15 (ref. ibid)]. Thermodynamic features testify on a special role of phonons [4-10, 14, 16-19]. Careful analysis of the TIST features and comparison with LIST made by us allows to separate, besides the structural features of ST systems and specific character of their chemical couplings, an apparent role of phonons [11, 12, 15, 20-26 (ref. ibid)]. Note also an importance of the following factors for ST: i) non-adiabaticity $[12,13,15,23]$; ii) vibronic interactions [12, 13, 15, 22-24]; iii) intrinsic anharmonicity $[12,13]$; iv) radiationless transitions $[12,13,20,25-27$ (ref. ibid)] and spin-orbital coupling [20, 23, 24, 28 (ref. ibid)]; v) contributions to formation of HS states of the terms with intermediate multiplicity (LIST), [4-10, 20 (ref. ibid)]. Note that special states with charge transfer in ligand complex are present and also a special role of $\pi$-coupling in SC process is observed [24, 29]. It is known that besides hydrogen couplings connecting both the polymeric chains and the complexes, $\pi$-coupling in SC systems, especially in polymers, intensifies the cooperativity and influences the hysteresis loop width $[14,30$, (ref. ibid)]. Therewith, polarized properties of molecules of the chemical elements and structural blocks of SC systems influence characteristically the formation of HS states and their stability [14, 20, (ref. ibid)]. In [20] by method of femtosecond transient infrared spectroscopy in combination with DFT (Density Functional Theory) quantum chemical calculations, the photoinduced ST of LS $(S=0)$ to HS $(S=2)$ type in iron (II) SC systems $[\mathrm{Fe}(\mathrm{btpa})]\left(\mathrm{PF}_{6}\right)_{2}$ and $\left[\mathrm{Fe}(\mathrm{b}(\mathrm{bdpa})]\left(\mathrm{PF}_{6}\right)_{2}\right.$ in solution has been studied at room temperature. These systems (under appropriate conditions) display an ultrafast consecutive states population with charge transfer, and also ultrafast population of the formed on iron (II)-complex ensemble of excited electronic states. The last displays through a conversion and fast relaxation of oscillations. Relaxation processes produce the HS state of the complex.

Important factors, which determine, in our opinion, a basic nature of ST in supramolecular SC compounds, first of all, organometallic systems, are considered in [20 ref. ibid] and similar works (see [4-10]), are, first of all, radiationless transitions stipulated by internal conversion and intersystem crossing, which are directly connected with mutual influence of electronic and phonon subsystems including spin-orbital coupling in the case of presence of electronic states with intermediate multiplicity $[20,26]$.

It is reasonable to suppose that for whole series of SC systems apparently a certain analogy of physical processes that take place at LIESST and TIST (in spite of different conditions of its behavior, $T=$ const and $T \neq$ const) is observed. This is possible as, at least, qualitatively the changing volumes of coordinated complexes of SC systems under such exciting factors, as light and temperature, are comparable, as it was noted above.

We suppose that above dependence of HS and LS states of the spin-crossover metal ion together with the features of stability formation of local HS states in the compounds studied are caused by structure peculiarity of the whole electronic shell of $3 d$ element including the core electrons. Formation and stability of spin contribution in the $3 d$ valence shell are only possible in the case of participation of the inner-shell and outer-shell electrons together with phonon subsystem and under influence of vibronic coupling and anharmonicity. For deeper complex study of short-range forces it is necessary to include the radiationless transitions and non-adiabaticity.

Note that the experiments supported by DFT calculations show that the space size variation of closed electron system and spin distribution in it are related closely not only for studied SC complexes with $3 d$ ions but for simplest compounds of iron group $[31,32]$. So, in $\mathrm{Fe}, \mathrm{Ni}, \mathrm{V}$ alike connection of magnetic order and lattice expansion/compression is observed. That is predetermined by a construction feature of its electronic shell and contribution of core electrons to chemical bond together with valence ones [32].

\section{Thermodynamic Aspects of SC}

In agreement with the thermal dependence of HS (LS) states of spin-active metal ion on volume, the TIST usually is considered as related with structural phase transitions (SPT) of reconstructive kind or PT of the first order. In absence of hysteresis it juxtaposited with SPT of distortional kind or PT of second order $[33,34]$. We think that taking into account a special physical properties displaying by ST structures when influenced by various disturbances, a TIST conception in itself as a certain kind of SPT is not true if not for any case but, at least, for majority organometallic compounds. In some cases, in is considered that TIST can combine the elements of both kinds of PT. It is obvious that both the phase conception and description of SC nature both including hysteresis and do not including one need, for any case of ST inducing, more complex approach. We believe that such an approach must be related to super-cooperative behavior of the system and mutual interdependence of interactions of its micro- and macrostates. 
We propose to describe ST from a point of view of socalled post-critical transformations (PCT) $[18,35,36]$. In our opinion, such an approach is valid for whole series of TIST compounds. In case of PIST and LIESST, additional investigations are needed.

In contrast to PT of conventional type, true PCT are purely continuous PT. According to $[35,36]$, post-critical range is, to some degree, a thermodynamic reservoir filled with a homogeneous mixture of fluctuating, competing with each other, turning in each other seeds of spin phases. Each of them tends to develop in true phase. However, being in the mixture they are in relatively stable dynamical equilibrium. PCT includes the range of maximal fluctuation development into over-critical space of changing thermodynamic parameters $(T, P, V)$. Range of maximal fluctuations is a curve of state passing through the points of isotherms bend and determining a range of reduced stability into over-critical space of isothermal sections of $P V T$-surface [37]. For example, for a system liquid-gas this curve consists of the bend points of isotherms in coordinate system of thermodynamic variables $(P, V)$ only $[35,36]$.

Any PT can be estimated most objectively in terms of thermodynamics of stability. Thus, heat capacity of TIST systems and their thermodynamic potentials are (in contrast to conventional PT treated as SPT) not simply continuous but smooth S-like (near transition point) curves without discontinuities which have exclusive peculiarity, namely, existence of bend points on the curves. That is typically just for PCT (Fig. 1, 2) [18, 35, 36].

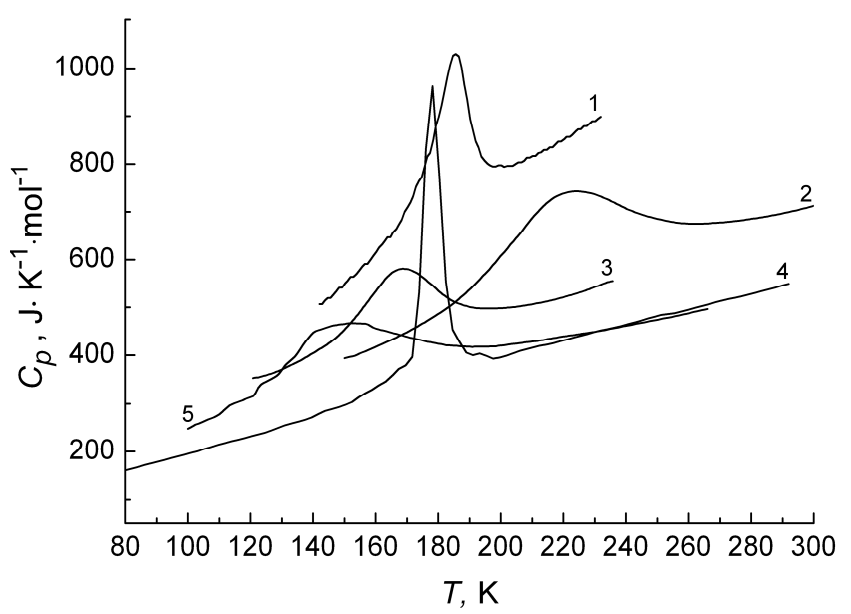

Fig. 1. Experimental dependences $C_{P}(T): 1-\left[\mathrm{Fe}(\text { hyptrz })_{3}\right] A_{2} \cdot \mathrm{H}_{2} \mathrm{O}, 2-$ $\left[\mathrm{Fe}(\mathrm{abpt})_{2}(\mathrm{NCSe})_{2}\right], 3-\left[\mathrm{Fe}(\mathrm{abpt})_{2}(\mathrm{NCS})_{2}\right], 4-\left[\mathrm{Fe}(\mathrm{phen})_{2}(\mathrm{NCS})_{2}\right], 5-[\mathrm{Fe}$ $\left(2-\mathrm{pic}_{3}\right]_{3} \mathrm{Cl}_{2} \cdot \mathrm{CH}_{3} \mathrm{OH}$.

Fig. 1 shows the results of calorimetric measurements for series of supramolecular compounds with temperatureinduced spin transition (TIST) of HS - LS kind: $\left[\mathrm{Fe}(\text { phen })_{2}(\mathrm{NCS})_{2}\right]$ with small hysteresis $[17,38]$; $[\mathrm{Fe}(2-$ pic) $\left.)_{3}\right] \mathrm{Cl}_{2} \cdot \mathrm{CH}_{3} \mathrm{OH}$ [17]; [Fe(abpt $\left.)_{2}(\mathrm{NCX})_{2}\right](\mathrm{X}=\mathrm{S}, \mathrm{Se})$ [39]; $\left[\mathrm{Fe}(\text { hyptrz })_{3}\right] \mathrm{A}_{2} \cdot \mathrm{H}_{2} \mathrm{O} \quad(\mathrm{A}=$ 4-chlorobenzenesulfonate $) \quad(1 \mathrm{D}$ polymer with hysteresis) [40]. Fig.2 demonstrates the reconstructed thermal dependences of entropy for the systems studied [22]. Fig. 3 shows temperature dependencies of entropy of water (for various pressures) which allow comparing a substance behavior in before-critical and postcritical states [36].

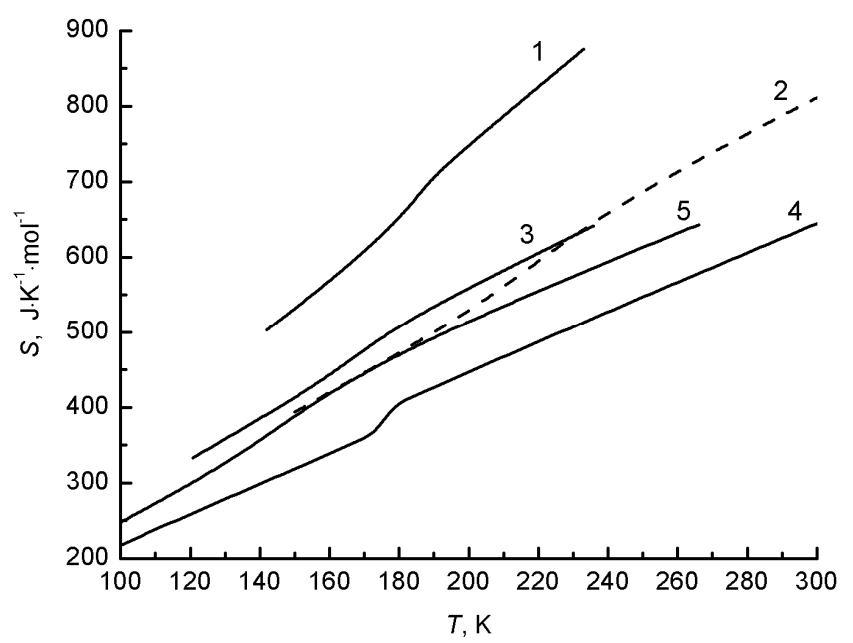

Fig. 2. Entropy dependence on temperature: $1-\left[\mathrm{Fe}(\text { hyptrz })_{3}\right] \mathrm{A}_{2} \cdot \mathrm{H}_{2} \mathrm{O}, 2-$ $\left[\mathrm{Fe}(\mathrm{abpt})_{2}(\mathrm{NCSe})_{2}\right], 3-\left[\mathrm{Fe}(\mathrm{abpt})_{2}(\mathrm{NCS})_{2}\right], 4-\left[\mathrm{Fe}(\text { phen })_{2}(\mathrm{NCS})_{2}\right], 5-[\mathrm{Fe}$ (2-pic) $\left.{ }_{3}\right] \mathrm{Cl}_{2} \cdot \mathrm{CH}_{3} \mathrm{OH}[18]$.

Comparison these data with Fig.2 allows us to make certain assumptions about the character of TIST for studied objects. Just a specific behavior of thermo-baric functional dependence of thermodynamic curves on $T$ and $P$, their continuity, smoothness, change of the slope and curvature, and above all, an existence of characteristic bend points reflect the main features of interactions in SC system, emphasizing their totally complex and cooperative behavior. Specific shape of the curves reflects a peculiarity of transformation of electronic-oscillatory degrees of freedom of the system, degree of their mixing, and features of cooperative coupling in the system. Points of bend (influction points on $\mathrm{C}_{\mathrm{p}}(\mathrm{T})$ curve) conditionally assign a PCT range with maximal development of fluctuations, cutting off on $C_{p}(\mathrm{~T})$ curve the corresponding segments with concavity and salience. Actually, fluctuation maximum is reached in the transition point $\mathrm{T}_{\mathrm{c}}=\mathrm{T}_{1 / 2}$ [4-10]. At the time point of bend $\mathrm{T}_{\max }$ (extremal point on $\mathrm{C}_{\mathrm{p}}\left(\mathrm{T}_{\max }\right)$ curve $-\mathrm{T}_{\max } \neq \mathrm{T}_{\mathrm{c}}$ - influction point on $\rho_{\mathrm{H}}\left(\mathrm{T}_{\mathrm{c}}\right)$ curve - "function distribution" (statistical weight $\rho_{\mathrm{H}} \leq 1$ ) of spin states HS phase) [10] can be equal to $\mathrm{T}_{1 / 2}$ symbolically only. Equality $\mathrm{T}_{\max }=\mathrm{T}_{\mathrm{c}}$ is not exact.

We emphasize that simultaneous existence both the salience and concavity distinguishes PCT from conventional PT. It is obviously that corresponding behavior of the slope and curvature of $C_{p}(T)$ curves on both sides from $T_{\mathrm{c}}$ reflects the peculiarities of the interaction in the system both in the case SPT and PCT.

It is convenient to describe the behavior of thermodynamic and mechanical functions of the system in terms of thermodynamics of stability [35, 36]. According to it, a necessary condition of stability of homogeneous system is a positivity stability determinant $\left(D_{\text {st }}\right)$ : 


$$
\begin{gathered}
D_{\text {st }}=\frac{\partial(T,-P)}{\partial(S, V)}>0 \text { and } \\
\left(\frac{\partial T}{\partial S}\right)_{V}=\frac{T}{C_{V}}>0,\left(-\frac{\partial P}{\partial V}\right)_{S}>0
\end{gathered}
$$

Stability coefficients (CS) entering expression for stability determinant are also positive. On the one hand, they can be presented as adiabatic coefficients of stability (ACS) $\left(\partial X_{i} / \partial x_{i}\right)_{x_{j}}$, and on the other hand, as isodynamic coefficients of stability (ICS) $\left(\partial X_{i} / \partial x_{i}\right)_{X_{j}}$, where $\mathrm{Xi}=\mathrm{T},-$ $\mathrm{P}, \ldots ; \mathrm{xi}=\mathrm{S}, \mathrm{V}, \ldots$ It is obviously that on basis of Jacobeans properties the ACS can be transformed into ICS (and vice versa). This allows representing Dst in convenient for analysis diagonal form:

$$
D_{\mathrm{st}}=\left(\frac{\partial T}{\partial S}\right)_{P}\left(-\frac{\partial P}{\partial V}\right)_{S}=\left(\frac{\partial T}{\partial S}\right)_{V}\left(-\frac{\partial P}{\partial V}\right)_{T}
$$

that is the product of ACS and ICS.

According to thermodynamics of stability method $[35,36]$, PCT are characterized by continuity both the stability coefficients and stability determinant defined by the first. According to the principles of thermodynamics of stability, PT in magnets and ferroelectrics should be ascribed as PCT systems, at least partially $[35,36]$. Temperature dependences both $D_{\text {st }}$ and CS for PCT systems, in which TIST can be realized (in contrast to analogue discontinuous dependences for PT of the first and second kind), are characterized by, first of all, continuity and positivity $[18,35,36]$. CS are equal to zero for real critical phenomena only.

Therewith, CS and $D_{\text {st }}$ dependences have clear minima at PCT, which are distant from abscissa axis in post-critical range $[18,36]$. These extrema are related to that range of "over-critical space" of changing substance parameters where maximum for this range comparative system instability relative to fluctuations (fluctuation maximum) $[18$, 35, 36].

Proposed there approach for describing of the completely compound series of TIST family, and possibly more wide kind of SC systems, meets with the explanations [41]. Particularly, in conception of ST as transition of orderdisorder kind TIST can be divided (for convenience) on three phase ranges. One of them is intermediate phase state of the substance, which is characterized by the same molecule concentration in HS and LS states in transition point. Total phase transformation is observed on the boundaries of temperature interval, i.e. at high and low temperatures.

\section{Conclusions}

It is reasonable shortly to stress that, according definition of PCT given in $[35,36]$, this is purely continuous and fluctuation transition (in contrast to conventional PT). It is assumed that the state of substance is always uniform. Transition at PCT occurs not in the point (as it takes place at
SPT in before-critical range) but in a certain interval of thermodynamic forces. A difference between the substance states near transition point in before-critical and post-critical ranges is seen more clearly in thermal dependences of entropy and thermal capacity changes (Fig. 1-3).

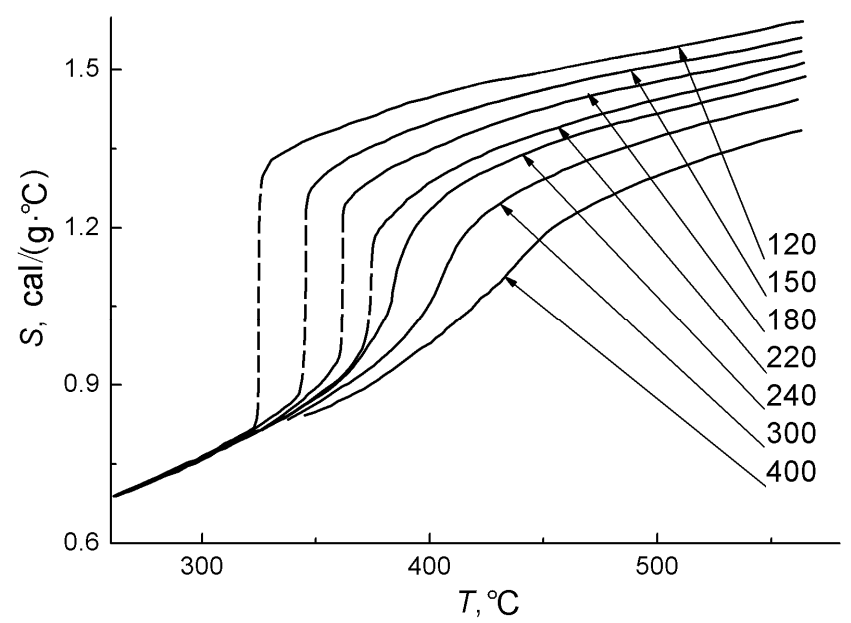

Fig. 3. Entropy change $S$ at sub-critical (discontinuous dependences), critical (curves between $220<P<240 \mathrm{~atm}$ isobars and close to vertical) and post-critical (continuous dependences) isobar transitions in water [36].

The curves are remarkable by characteristic changes of the slope, curvature and existence of special parts containing saliencies/concavities, that, as a matter of fact, corresponds to different nature of interactions in the systems with appropriate PT kind. So, conventional PT (before-critical PT) is characterized by monotonic change of $C_{p}(T)$ slope for both sides from $T_{\mathrm{c}}$ with the same kind of concavity, while the PCT is characterized by Gauss-like curve shape with distinctive transition from salience to concavity and influction points bounding special fluctuaton regions.

Authors of the monographs [35, 36] believe that it is possible to attribute to continuous fluctuation transitions not only PCT discovered by Andrews in systems liquid-gas [36, 37] but also many transitions in crystalline polymers, quartz, ferromagnets and ferroelectrics.

SC phenomenon observed in complex supramolecular compounds with ions of transition metals of iron group as spin-crossover centers reveals, owing to its continuity, a whole set of phenomena caused by dynamical conditional equilibrium of a complex quantum-mechanical micro- and macroscopic interactions, which are competing and interdependent. A nature of these direct and indirect connections in its cooperative basis is the links of the same succession with biological objects including coordination complexes with $\mathrm{Fe}$ (II) centers (hemoglobin, etc.). In this sense, TIST demonstrates a full analogy with S-like kinetics of oxygenation observed in hemoglobin [26]. This stresses a currency of cooperativity in supramolecular compounds.

We believe that at studying of SC phenomena in metalcontaining compounds a maximal attention should be paid on phonons, peculiarities of vibronic couplings and anharmonicity, polarization effects, complex multilevel role of $\pi$-coupling, participation of states with intermediate 
multiplicity and spin-orbital interaction, radiationless transitions, non-adiabaticity. In addition, it is necessary to ground and use a special role of core electrons of spincrossover metal ions, taking part in all physical processes observed in ST compounds. Also an active role in these processes of hydrogen and hydrogen-like chemical bonds including bridges connecting the complexes should be noted. These bonds are not only components of packing effect, but take part in formation and stabilization of cooperative coupling. Particularly, they together with frame electrons, directly take part in formation of distinctive domain structures and in formation of hysteresis which in complex compounds has own special peculiarities.

In conclusion, authors hope that the proposed nonconventional approach to study of complex compounds, particularly SC, considered from PCT position would serve as catalyst for analyze of actual problems and solving of key questions of ST mechanism.

\section{References}

[1] O. Kahn, Molecular magnetism, VCH Publishers, Inc. (1993).

[2] P. Gütlich, A. Hauser, H. Spiering, Angew. Chem. 33, 2024 (1994).

[3] P. Gütlich, Y. Garcia, H.A. Goodwin, Chem. Soc. Rev., 29, 419 (2000).

[4] .P. Gütlich, P. J. van Koningsbruggen, F. Renz, Structure and Bonding 107, 27 (2004); A.B. Gaspar, M. Seredyuk, P. Gütlich, J. Mol. Struc., 924-926, 9 (2009).

[5] V. Legrand, Présentée pour l'obtention du titre deDocteur de l'Université Henri Poincaré, Nancy-Ien Physique et Chimie de la Matière et des Matériaux. (2005).

[6] G.G.Levchenko, A.V.Khristov, Molecular magnetism: high spin - low spin phase transitions. "Noulidge" Publishers, Donetsk, Ukraine (2010).

[7] A.Bousseksou, G.Molnar, L.Salmona and W.Nicolazzi, Chem. Soc. Rev., v. 40, p. 3313 (2011).

[8] J.Linares, E.Cojovi and Y.Garcia, Sensors, v. 12, p. 4479 (2012).

[9] P. Gütlich, Eur. J. Inorg. Chem., p. 581 - 891 (2013).

[10] G.Levchenko, A.Khristov, V.Kuznetsova, V.Shelest. Journal of Physics and Chemistry of Solid, v. 75, № 8, p.966 - 971 (2014)

[11] K. L.Ronayne, H. Paulsen, A. Höfer, A.C. Dennis, J.A. Wolny, A.I. Chumakov, V. Schünemann, H. Winkler, H. Spiering, A. Bousseksou, P. Gütlich, A.X. Trautwein, J. McGarvey, Phys. Chem. Chem. Phys., 8, 4685, (2006).

[12] I.B.Bersuker. Electronic structure and properties of coordination compounds, "Khimiya" Publishers, Leningrad (1976); Jan-Teller effect and vibronic interactions in modern chemistry, "Nauka" Publishers, Moscow (1987); Modern aspects of the Jahn-Teller effect theory and applications to molecular problems, Chem. Rev., 101, 1067 (2001).
[13] S.V.Volkov, V.A.Zasukha, Quantum chemistry of coordination condensed systems, "Naukova dumka" Publishers, Kiev (1985).

[14] V. Martinez, A.B. Gaspar, M.C. Muñoz, G.V. Bukin, G. Levchenko, J.A. Real, Chem. Eur. J., 15, 10960 (2009).

[15] V. Gnezdilov, P. Lemmens, P. Sceib, M. Ghosh, Yu. G. Paskevich, H. Paulsen, V. Schünemann, J.A. Wolny, G. Agusti, J.A. Real, Phys. Rev. B, 79, 045122 (2009).

[16] Sorai M., Ensling J., Gütlich P. // Chem.Phys. - 1976. - V. 18. - P. 199-209.

[17] M. Sorai, Bull. Chem. Soc. Jpn., 74, 2223 (2001).

[18] V.V.Shelest, A.V.Christov. Proceedings of International conference "Actual problems of solid state physics" Minsk, Belarus, Abstr., P.243 (2009).

[19] J.A. Rodríguez-Velamazán, M. Castro, E. Palacios, R. Burriel, J.S. Costa, J.F. Létard, Chem. Phys. Lett., 435, 358 (2007).

[20] M.M.N. Wolf, R. Groß, C. Schumann, J.A. Wolny, V. Schünemann, A. Døssong, H. Paulsen, J.J. McGarvey, R. Diller, Phys. Chem. Chem. Phys., 10, 4264 (2008).

[21] Y. Konishi, N. Tokoro, M. Nishino, S. Miyashita, Phys. Rev. Lett. PRL 100, 067206 (2008).

[22] K. Boukheddaden, M. Nishino, S. Miyashita, Phys. Rev. Lett., PRL 100, 177206 (2008).

[23] N.F.Stepanov, Quantum mechanics and quantum physics. "Moscow state university Publishers", Moscow (2001).

[24] C. Day, J. Selbin, Theoretical inorganic chemistry, New York - Amsterdam - London, «Reinhold Book Corporation» (1962).

[25] S. Klokishner, J. Linares, F. Varret, J. Phys.: Condens. Matter, 13, 595 (2001).

[26] Structure and bonding. Volume 2, edited by C.K. Jørgensen, J.B. Nielands, R.S. Nyholm, D. Reinen, R.J.P. Williams, "Springer - Verlag" Berlin - Heidelberg - New York (1967).

[27] G.A. Razuvaev, B.G. Gribov, G.A. Domracheev, B.A. Solomatin, Metallo-organic compounds in electronics, "Nauka" Publishers, Moscow (1972).

[28] V.I.Shipilov, V.A.Fedirko, V.V.Zelentsov, V.M.Mokshin. Sov. Solid State Phys., v.21, No 12, P.3553 (1979).

[29] E. Cartmell, G.W.A. Fowles, Valency and molecular structure, Butterworths (1978).

[30] G. Dupouy, M. Marchivie, S. Triki, J. Sala-Pala, J.-Y. Salaün, C.J. Gómez-García, P. Guionneau, Inorg. Chem., 47, 8921 (2008).

[31] John C. Slater, The self-cosistent field for molecules and solids, Москва, «Mc Graw-Hill Book Co.» New York - St. Louis - San Francisco (1974).

[32] Theory of the inhomogeneous electron gas, edited by S. Lundqvist, N.H. March, "Plenum Press", New York and London (1983).

[33] Sov. Solis State Phys. Encyclopedia, "Naukova dumka" Publishers, Kiev, VV.1, 2 (1998). 
[34] Bruce A.D., Cowley R.A., Structure phase transitions, London (1981).

[35] I.P.Bazarov, Thermodynamics, "Visshaya shkola" Publishers, Moscow (1991).

[36] V.K.Semenchenko, Selected chapters of theoretical physics, "Prosveschenie" Publishers, Moscow (1966).

[37] E. Stanley, Introduction to phase transitions and critical phenomena, «Clarendon Press», Oxford (1971).
[38] A. Bousseksou, N. Negre, M. Goiran, L. Salmon, J.-P. Tuchagues, M.-L. Boillot, K. Boukheddaden, F. Varret. Eur. Phys. J. B, 13, 451 (2004).

[39] N. Moliner, C. Muñoz, S. Létard, J-F. Létard et al., Inorg. Chem. Acta, 291, 279 (1999).

[40] Y. Garcia, V. Ksenofontov, G. Levchenko, P. Gütlich, J. Mater Chem., 10, 2274 (2000).

[41] D. Chernyshov, M. Hostettler, K. Tömroos, H.S. Bürgi, Angew. Chem. Int. End. 42, 3825 (2003). 